\title{
Atrial Macroreentry in Congenital Heart Disease
}

\author{
Darragh J. Twomey, Prashanthan Sanders and Kurt C. Roberts-Thomson*
}

Centre for Heart Rhythm Disorders, University of Adelaide and Royal Adelaide Hospital, Adelaide, Australia

\begin{abstract}
Macroreentrant atrial tachycardia is a common complication following surgery for congenital heart disease (CHD), and is often highly symptomatic with potentially significant hamodynamic consequences. Medical management is often unsuccessful, requiring the use of invasive procedures. Cavotricuspid isthmus dependent flutter is the most common circuit but atypical circuits also exist, involving sites of surgical intervention or areas of scar related to abnormal hemodynamics. Ablation can be technically challenging, due to complex anatomy, and difficulty with catheter stability. A thorough assessment of the patients status and pre-catheter ablation planning is critical to successfully managing these patients.
\end{abstract}

Keywords: Congenital heart disease, supraventricular tachycardia, catheter ablation.

\section{INTRODUCTION}

Significant congenital heart disease (CHD) occurs in 6 of every 1000 live births [1]. In adulthood, arrhythmias are often problematic in these patients and present significant therapeutic challenges. These patients have distorted anatomy, hemodynamic stress and often hypoxia, and conduction system abnormalities, leading to both brady and tachyarrhythmias. While many forms of atrial tachyarrhythmias have been described, including AV nodal reentry, focal atrial tachycardia [2] focal junctional tachycardia [3] and AF, macroreentry is the most common form of tachyarrhythmia in this group. These arrhythmias are termed 'intra-atrial reentrant tachycardias' (IART) and are due to reentry around an anatomic obstacle, at least 2 centimeters in diameter [4]. In this review we will focus on this arrhythmia in the common forms of CHD.

\section{GENERAL CONSIDERATIONS}

IART occurs in all forms of CHD, from atrial septal defect and Tetralogy of Fallot repair, to more complex disease, such as following the Mustard and Senning procedures for transposition of the great arteries and the Fontan procedure in patients with a univentricular circulation due to tricuspid atresia or a double inlet left ventricle. Arrhythmia most often presents in adulthood, years after surgery and more complex anatomical lesions are associated with a higher risk of developing this complication. In conjunction with the precarious haemodynamic status often seen in complex CHD, these arrhythmias are often poorly tolerated, and significant morbidity can occur. It is important to recognize that arrhythmias may represent a change in hemodynamics and should prompt reassessment of the patient's status.

\footnotetext{
*Address correspondence to this author at the Centre for Heart Rhythm Disorders, Level 5, McEwin Building, Royal Adelaide Hospital, North Terrace, Adelaide, SA 5000, Australia; Tel: +61 88222 2723; Fax: +61 8 8222 2722; E-mail: kurt.roberts-thomson@adelaide.edu.au
}

\section{THERAPEUTIC OPTIONS}

The options for treatment in patients with IART and CHD include antiarrhythmic medications, catheter ablation, pacing and surgery.

\section{MEDICAL MANAGEMENT}

There are few reports of successful medical therapy for IART. Published ablation data often includes details of failed medication, but significant bias exists as these groups are selected for ablation due to drug resistance. In general, the principles for antiarrhythmic therapy are as for other forms of heart disease. Therapeutic regimens used included betablockers, calcium channel antagonists, class Ia agents, class III agents and digoxin, administered alone or in combination [5]. One study, prior to the advent of ablation for IART, probed 19 patients and reported successful arrhythmia suppression with class 1a agents (plus beta blockers or digoxin) in 6 patients, with 11 requiring amiodarone and 2 undergoing pacemaker implantation. Beta blockers or calcium channel antagonists alone did not maintain sinus rhythm in any patient [6]. Dofetilide has been used in patients with congenital heart disease and, in a multicenter study, demonstrated initial success rates of $85 \%$ with maintenance of sinus rhythm in the long term of 55\% [7]. Two patients had torsades de points at high dose and the authors suggest halving the dose in these patients. Amiodarone has been considered the most effective medication, but its side effects limit its use in young patients.

One of the concerns with atrial arrhythmias in this population is the potential risk of thromboembolic complications. Indeed, there is growing evidence to suggest that increased atrial rates alone may be sufficient to confer a thromboembolic risk. A study examining transesophageal echocardiograms in non-anticoagulated patients with congenital heart disease and regular narrow complex tachycardia revealed a high incidence of intra-atrial thrombus (42\%), [8] suggesting anticoagulation is generally indicated. 


\section{ABLATION}

Adequate medical management of IART is frequently not possible [9] and an invasive strategy is often required. Ablation of the reentry circuits is technically challenging and reported success rates are lower than standard atrial arrhythmias, with increasingly complex CHD associated with lower rates of ablation success. Multiple circuits may exist, which can involve both artificial (suture lines, prosthetic material) and natural (crista, valve annuli and vena cavae) regions of conduction block. Complex anatomy is associated with increasingly complex circuits and the use of electroanatomical mapping systems, potentially in conjunction with preprocedural MRI or CT assessment of cardiac structure, allows accurate characterization of these circuits and regions of conduction block, and may improve outcomes [10,11]. The distorted anatomy and complex circuits make the $p$ wave morphology difficult to interpret and thus careful activation and entrainment mapping are critical to determining the mechanism and location of the arrhythmia. These patients have particular issues complicating catheter ablation, particularly with regard to the vascular access and distorted anatomy. In all cases, knowledge of the surgical incisions and operation is important in planning the ablation procedure, and the use of technologies such as Remote Magnetic Mapping may improve catheter stability $[12,13]$.

\section{PACEMAKER IMPLANTATION}

In cases resistant to ablation therapy, pacing may be considered as an alternative therapy, and is often indicated for coexistent sinus node disease. Pacemakers capable of antitachycardia pacing have in some series been found to be useful when the arrhythmia demonstrates pace terminability [14]. although anatomical variations can offer a significant technical challenge to the implanter. Following pacemaker insertion, medical rate control or catheter ablation of the AV node can provide symptomatic improvement.

\section{SURGICAL INTERVENTION}

Rarely has further surgical intervention been advocated as often this is at a greater risk to the individual. However, there are some series that have described successful intervention in highly resistant cases, although this may only be appropriate if further additional surgery is warranted on hemodynamic grounds [15] as operative mortality can be as high as $10 \%[16]$.

\section{ATRIAL SEPTAL DEFECTS (ASD)}

The incidence of IART can be difficult to estimate as many studies examining its prevalence do not distinguish between IART and other atrial arrhythmias. One early study showed the incidence of atrial flutter to be as high as $18 \%$ over a mean of only 2.3 years post ASD repair [17]. Another found all supraventricular tachycardias occurred in only 7\% in a cohort of 135 patients over a mean follow up of 15 years [18]. It is recognized that prior to surgery, the dilated right and left atria show electroanatomic remodeling characterized by prolonged refractory periods, conduction slowing and reduced voltage, predisposing to re-entrant arrhythmias [19, 20]. ASD closure, using a transcatheter device or surgically, appears to reduce the incidence of arrhythmia [21-23].

ASD repair usually involves a lateral right atrial incision prior to closure of the defect with direct closure, or the use of a pericardial or Dacron patch.

The most common circuit is cavotricuspid isthmus dependent flutter. In patients who have undergone surgery, reentry around the atriotomy incision is also observed (Fig. 1). Several studies have demonstrated the coexistance of two distinct macro reentrant circuits, or 'dual-loop' re-entry [2426]. One described 5 patients following ASD repair. All had CTI-dependant atrial flutter, with three having additional reentry around the atriotomy scar [24]. Shah et al. also reported 5 cases of post ASD repair right atrial flutter present-

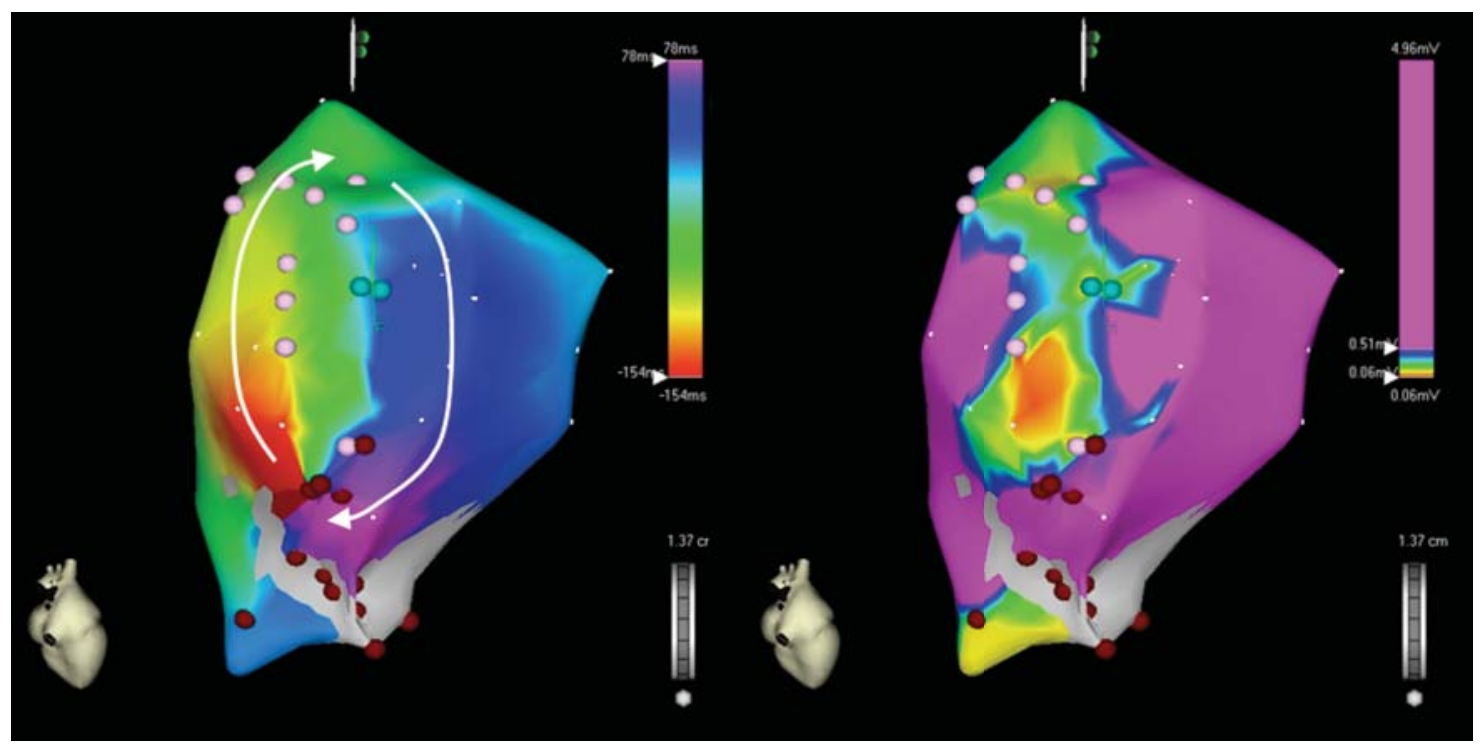

Fig. (1). This figure shows electroanatomic maps of the lateral right atrium in a patient with IART post surgical ASD repair. The left map is an activation map demonstrating a clockwise re-entrant circuit. The right map is a voltage map demonstrating the region of low voltage, colored in red and green, on the lateral wall which corresponds to the atriotomy incision. This incision is the central obstacle around which the tachycardia circuit revolves. Catheter ablation (red dots) between the region of low voltage and the inferior vena cava terminated tachycardia. 
ing with an ECG pattern suggestive of typical flutter. Following electroanatomical mapping, it was found that two circuits were present in each patient. After ablation in the CTI, the ECG changed abruptly, predominantly with positive $\mathrm{p}$ waves in the inferior leads, but a mean change in cycle length of only 13 milliseconds and no intervening pause. In one patient, the surface ECG was unchanged except for a change in cycle length. The second tachycardia was circuiting the atriotomy scar and a second line of ablation was then required between the atriotomy scar and the IVC, or in one case, the scar and tricuspid annulus [25]. This highlights the importance of monitoring the ECG morphology and activation patterns closely during ablation. In some cases there is a conduction channel traversing the free wall scar that can sustain the free wall tachycardia. Focal ablation at this site can abolish tachycardia. Kalman et al., following the exclusion of cases of typical flutter, examined 9 patients with 10 IARTs. The majority of these were incisional tachycardias on the right atrial free wall but the authors also described a case of reentry around the septal patch. This required ablation between the patch and the tricuspid annulus. Left sided IART is rare, but can occur (Fig. 2).

Snowdon et al. recently published techniques for assessing ablation line integrity in right free wall flutter. Atriotomy to IVC lines was assessed by pacing the ablation catheter, placed posterior to the line in the low lateral RA, and recording the timing and activation sequence of electrograms produced by a 20-pole catheter positioned between the scar and the tricuspid valve. Similarly atriotomy to TV lines can be assessed by pacing inferior to the line [27].

Flutter wave morphology can be useful prior to EP assessment. In one study of 16 patients, all patients with typical morphology had an isthmus dependant circuit and no patient with atypical morphology was found to have only a CTI-dependant arrhythmia [24]. However, a significant proportion of both typical and atypical flutter patterns had more than one circuit, demonstrating the lack of specificity from ECG assessment alone.

\section{TETRALOGY OF FALLOT}

In a large cohort of 793 patients, the incidence of atrial flutter or fibrillation over a 10 year period was only $7 \%$ [28]. A recent multi-centre study found $11.5 \%$ of patients with corrected Tetralogy of Fallot developed IART, although this did include a small number of patients with pulmonary atresia. Analysis of this cohort also found IART was more prevalent in patients with RA enlargement, hypertension and those who had more than one surgical procedure [29]. RoosHesselink and colleagues found all patients with atrial arrhythmias to have right atrial and right ventricular enlargement on echocardiography [30].

Surgery for Tetralogy of Fallot involves patch closure of the ventricular septal defect and enlargement of the right ventricular outflow tract. Access has generally been via the right ventricle, which can predispose to ventricular arrhythmias, but a trans-atrial approach is now mainly used [28].

The atrial incision for a Tetralogy of Fallot repair is similar to that used in patients to repair an ASD and therefore the arrhythmias are similar. Nakagawa et al. described 3 patients with IART and Tetralogy of Fallot. All had a lower atrial scar or line of double potentials, confluent with the IVC and a more superior, anterior scar. Successful focal ablation was performed between these 2 areas in all cases [31]. Triedman and colleagues found successful sites to be at the inferomedial RA in both patients with Tetralogy of Fallot, with one

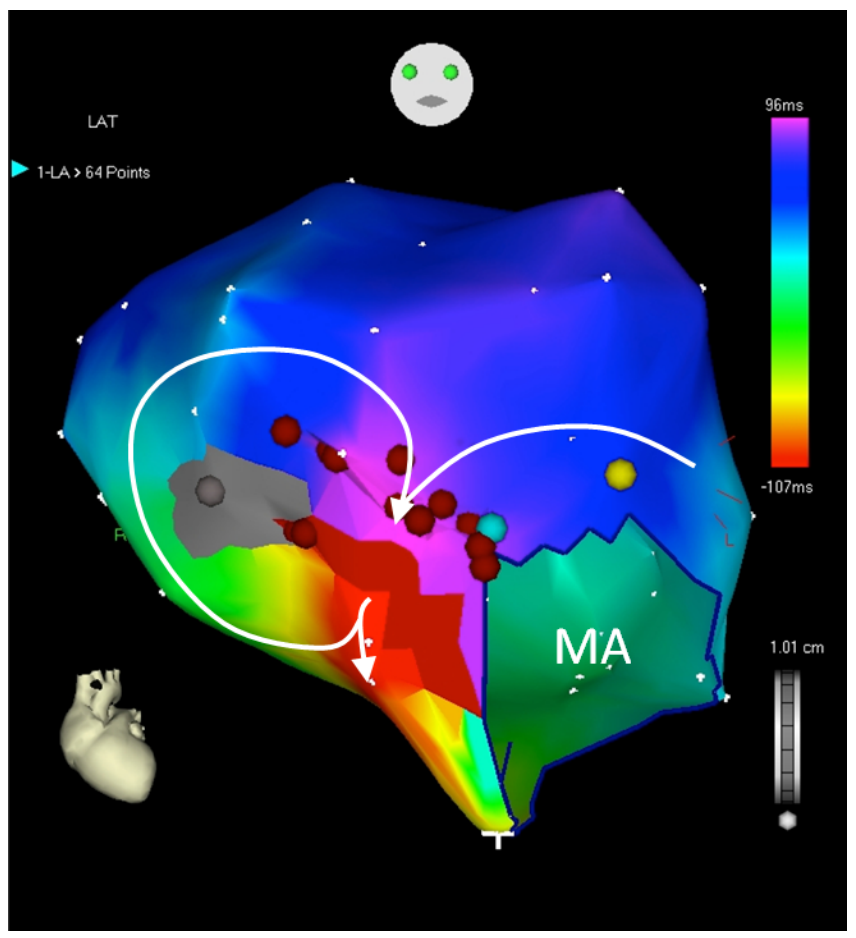

Fig. (2). This figure is an electroanatomic map of the left atrium, in the anterior view, in a patient post ASD repair. This activation map demonstrates the presence of dual loop reentry around the mitral annulus (MA) and the ASD patch (shown in grey). Catheter ablation (red dots) between the patch and the mitral annulus terminated tachycardia. 
also requiring ablation at the RA-SVC junction [5]. Another study reported successful ablation in the posterior CTI in 3 patients [32].

\section{EBSTEIN'S ANOMALY}

Up to one third of patients with Ebstein's anomaly have an accessory pathway. These are mainly right sided and often multiple and most arrhythmias are related to this. The abnormal tricuspid valve can require surgical repair, along with concomitant congenital lesions, increasing the probability of macroreentrant circuits [33].

Surgical intervention for tricuspid regurgitation or additional lesions predisposes to IART. Right atrial dilatation is common and can promote CTI dependant arrhythmia. Catheter stability can be difficult on the abnormal tricuspid isthmus. In the largest tachyarrhythmia study of this group, comprising 32 patients, there were 5 instances of CTI dependant flutter and 5 of other IARTs, which were found to consist of multiple circuits in two [33].

\section{TRANSPOSITION OF THE GREAT ARTERIES}

Transposition of the great arteries is the commonest cause of atrial flutter in the young [9]. The incidence of atrial flutter following Mustard surgery was $25 \%$ over a mean follow up period of 3 years in one early study [17]. Another found a $40 \%$ incidence of all supraventricular tachycardias, including AF, over a mean of 4.5 years. This study also found a weak association between supraventricular tachyarrhythmia and sudden death [34]. A third included all regular supraventricular tachycardias and found the incidence to be 6,12 and $17 \%$ at 1,3 and 5 years post-op, with older age of operation, the need for valve replacement and older surgical techniques being associated with a higher risk of SVT [35].

Atrial switch procedures for Transposition of the Great Arteries involve the creation of an intra-atrial baffle to divert deoxygenated systemic venous blood to the mitral valve, left ventricle (LV), pulmonary artery (PA) and pulmonary circulation and oxygenated blood, returning from the lungs, to the tricuspid valve, right ventricle, (RV) aorta and systemic circulation. The Senning procedure involves creation of the baffle solely from atrial tissue whereas the Mustard procedure uses a single patch made of pericardial tissue or Dacron, following excision of the atrial septum (Fig. 3). Significant anatomical variation can occur. For example, during the Mustard procedure, the coronary sinus is generally kept on the pulmonary venous side, but a suture line can be placed anterior to it, keeping it on the systemic venous side. These variations highlight the need for careful review of operative notes prior to undertaking catheter ablation.

Femoral artery access is often required to allow mapping of the pulmonary venous atrium retrogradely via the aorta. The tortuosity of this route can make catheter manipulation and stability during ablation difficult.

In Mustard patients, the baffle suture line can act as an area of conduction block. Senning patients have more complex surgical anatomy, one study described a number of lines of conduction block in detail: 1) the suture line where the atrial septal flap forms a roof over the pulmonary veins; 2) the edge of the atrial septal remnant; 3) the suture line where the right atrial free wall attaches to the atrial septal remnant to form the roof over the systemic venous tube and 4) the suture line where the rightward most section of the pulmonary venous atrium is attached to the remaining right atrial free wall [2]. 10 circuits were identified in 9 Mustard patients. Of these, 9 involved the isthmus between the tricuspid annulus and IVC but successful ablation was only possible in 6. In all cases, only the medial isthmus could be accessed from the systemic venous side, the lateral isthmus was always found on the pulmonary venous side, requiring femoral arterial access, negotiation through the right ventricle, and prolapse of the ablation catheter across the tricuspid valve into the pulmonary venous atrium. In a study of 14 Mustard and Senning circuits, Collins and colleagues showed the valve annulus-IVC isthmus to be the successful ablation site in 8 , with 6 of these requiring retrograde access to the pulmonary venous aspect of the baffle. The remaining 6 circuits were ablated in the lateral RA wall [36]. One study of 4 pa-
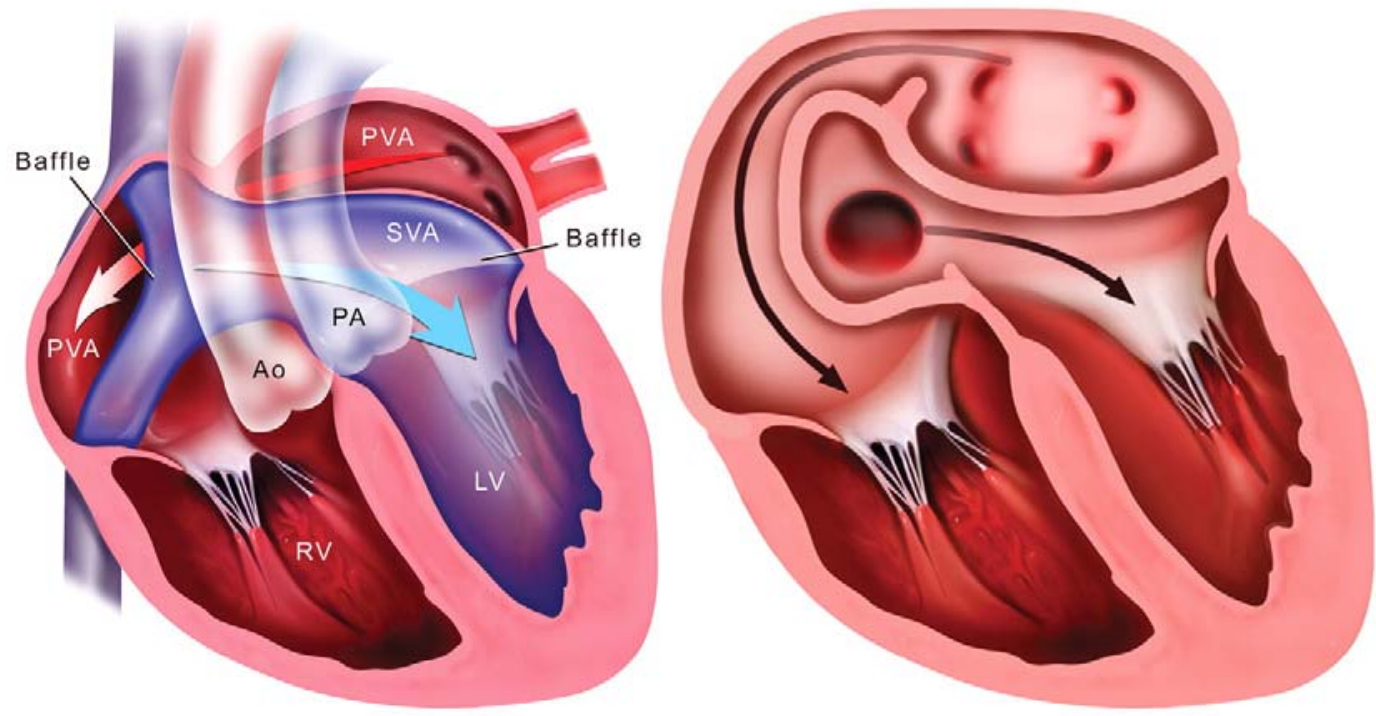

Fig. (3). This figure shows the surgical conduits created by the Senning procedure, with a cut-away illustration on the right. (Reproduced with permission - Gaca AM et al. Radiology. 2008; 247:617-631). 
tients with 5 IARTs outside the CTI, demonstrated the site of successful ablation to be between the baffle and coronary sinus in 2, the baffle and tricuspid annulus in 1, the atriotomy and tricuspid annulus in 1 and the low posterior systemic venous atrium in 1 [37]. Chan et al. described successful ablation locations in 3 Mustard patients, all of which were in the systemic venous atrium, 2 at the coronary sinus ostium, and one in the low atrium [32]. In contrast, the study by Yap et al. demonstrated that only $29 \%$ of IARTs were CTI-dependant in post-Mustard patients and that successful RFA was significantly less likely, when compared with other CHD [10].

\section{UNIVENTRICULAR CIRCULATION}

Patients having undergone the Fontan procedure have a high incidence of atrial arrhythmias. In one study it occurred in $22 \%$ of patients [38], in another the incidence was $6 \%$ at 1 year and $17 \%$ at 5 years [35]. Takahashi et al. studied patients undergoing Fontan conversion surgery and found IART in $25 \%$, even prior to surgery [39].

Patients with univentricular circulation usually undergo the Fontan procedure in two stages. The first, also known as the bidirectional Glenn procedure, connects the superior vena cava to the right pulmonary artery. The inferior vena cava is connected to the pulmonary artery during the the second stage, also known as Fontan completion. Variations of the procedure occur, the classic Fontan connected the right atrium to the PA via a conduit; a widely used modification of this anastomosed the RA directly to the PA. More recently, an internal or external conduit has been used to connect the IVC to the PA, to create a total cavopulmonary

\section{Panel A)}

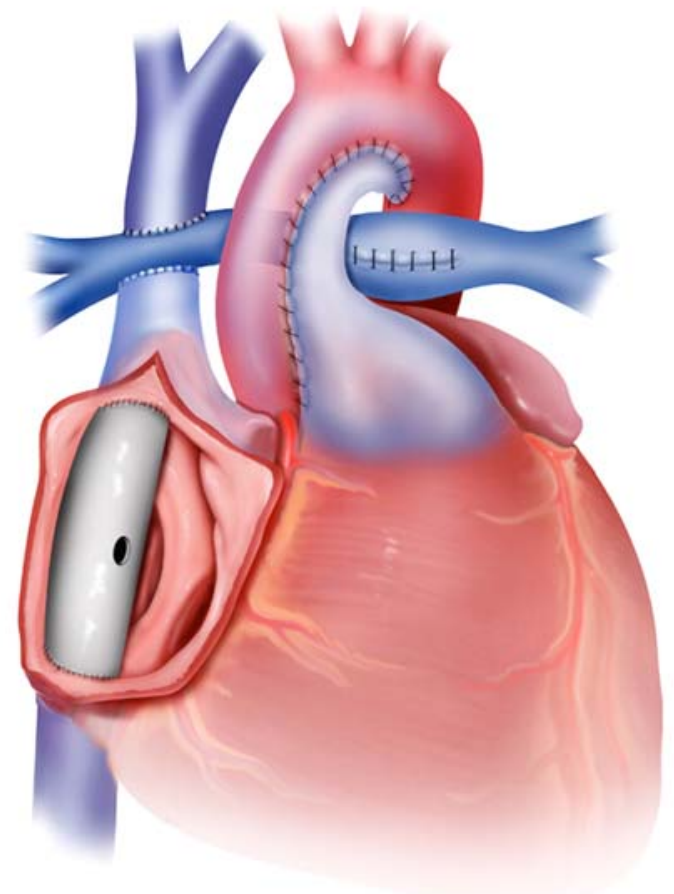

connection [40]. (Fig. 4). The highly complex anatomy and surgical intervention predisposes to significant atrial scarring.

The Fontan circulation is often associated with circuits that are neither perivalvular nor incisional, as shown in a study by Nakagawa and colleagues [31]. The scarring can be variable and thus the circuits also show a degree of variability between individuals (Fig. 5). Despite this, focal ablation of channels between areas of block successfully terminated arrhythmias in 5 of these patients. Several studies have highlighted the complexity of ablation in Fontan patients. One described 4 patients; two were successfully ablated by connecting the Fontan circuit and the TA in one and the atriotomy in the other. Successful ablation was not possible in the remaining two cases [37]. Another described both successful and unsuccessful sites in 6 patients. Two of these were successful at the SVC junction, one at the Fontan anastomosis, and one connected the anastomosis and the SVC. Three of these patients had further arrhythmic circuits; attempted ablation in the inferior RA was unsuccessful in all cases. One patient had a circuit which could not be ablated in the inferomedial RA and the final patient had 5 distinct circuits, all of which were successfully ablated [5]. Mandapati et al. studied 37 IART circuits in 22 Fontan patients and found all to be CTI dependant, with twelve revolving around the IVC, and the remainder being periannular [41]. A large study, including 40 patients, showed $53 \%$ of successful ablation sites were at the lateral RA, with $21 \%$ on the anterior wall, and only $15 \%$ involved the cavotricuspid isthmus [36]. In a large series of patients with CHD and IART, Yap et al. determined that patients with Fontan palliation were significantly more likely to

Panel B)

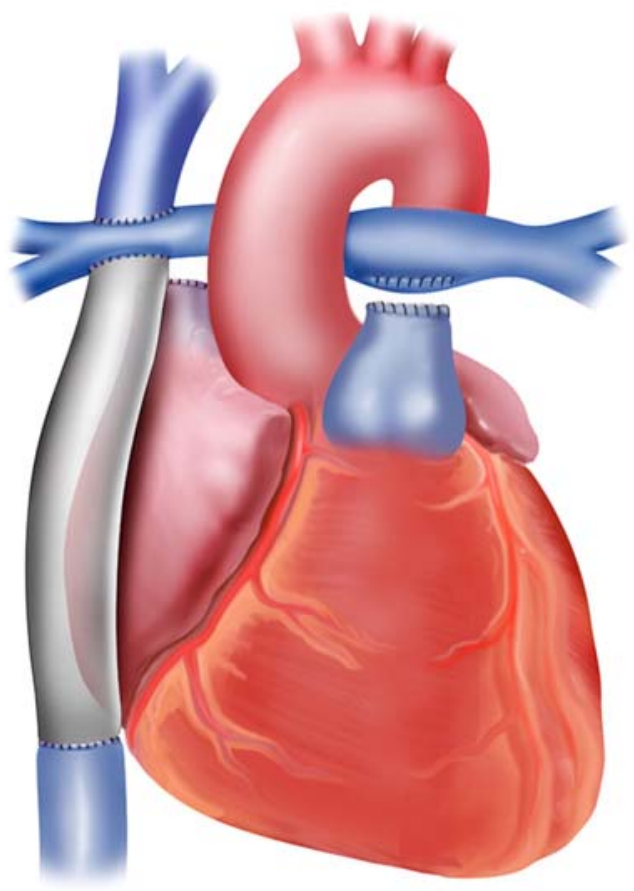

Fig. (4). This figure illustrates (A) the fenestrated Fontan lateral tunnel and (B) Fontan extracardiac conduit. (Reproduced with permission Gaca AM et al. Radiology 2008; 247: 617-31). 


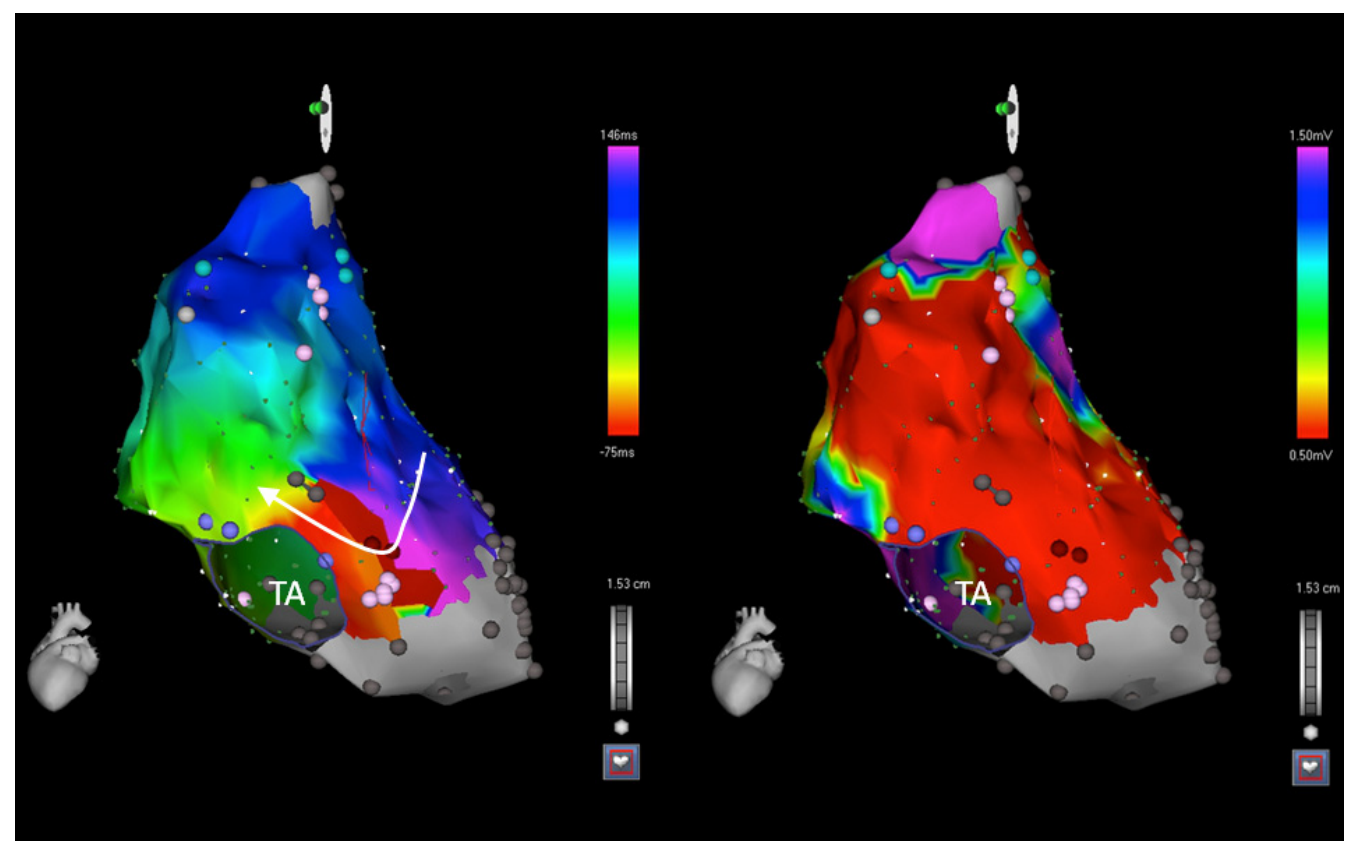

Fig. (5). This figure shows electroanatomic maps in a patient with IART post Fontan. The right voltage map demonstrates the large region of low voltage, shown in red, in this chamber. The left activation map demonstrates activation through the region of low voltage in the posteroseptal aspect of the right atrium. Catheter ablation (red dots) at this site terminated tachycardia. TA $=$ tricuspid annulus.

have a non-CTI circuit (79\%) when compared with other CHD patients (51\%).

\section{OUTCOMES}

In the largest published series, the overall acute success rate in 193 ablation procedures was $63 \%$, ranging from $43 \%$ in post-Mustard patients, to $76 \%$ in those post ASD closure (Fig. 6) [10]. Despite $79 \%$ of those successfully ablated remaining free of arrhythmia at 6 months, at 4 years this had dropped to $49 \%$, with Fontan patients showing a marked reduction in success rate, of only $15 \%$. At repeat study, the site of successful ablation was different to the original site in $62 \%$. The strongest predictor of recurrence was a previous Fontan operation (HR 2.18) along with increasing age. Triedman et al. reported the efficacy of RFA in 55 cases of IART and found recurrence was most likely to occur with 6 months of the procedure. $40 \%$ of patients were free of arrhythmia at 3 years [42]. In a later study, the predictors of a favorable outcome were determined by logistic regression and found to be the use of modern technology (electroanatomical mapping and irrigated ablation catheters), nonFontan physiology and fewer IART circuits [11].

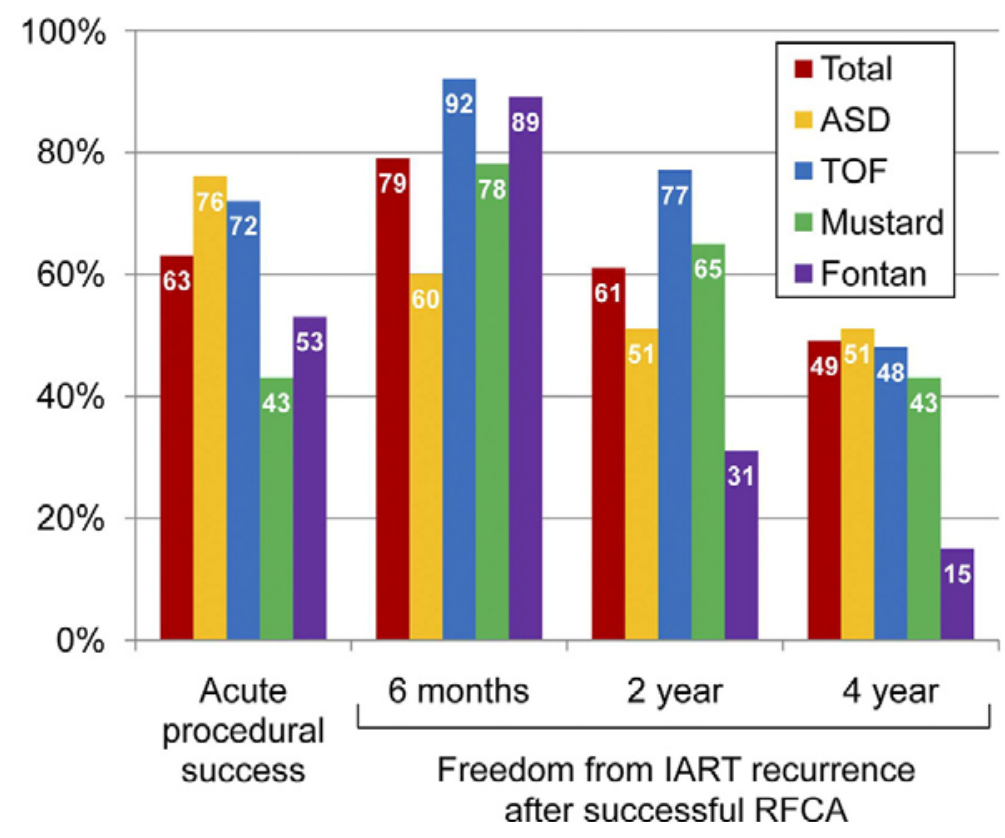

Fig. (6). This graph shows the acute and long term procedural success rates in different groups of surgically corrected CHD. (Reproduced with permission - Yap et al. J Am Coll Cardiol 2010; 56: 1589-96). 


\section{CONCLUSION}

IART in patients with surgically corrected congenital heart disease is uncommon in clinical practice but is prevalent in this small group of patients. Medical management is often unsuccessful, requiring the use of invasive procedures. Cavotricuspid isthmus dependent flutter is the most common arrhythmia but the existence of multiple circuits is common. In patients with increasingly complex anatomy, the presence of complex circuits at other sites becomes more frequent and success rates are lower. A thorough assessment of the patient's status and pre-catheter ablation planning is critical.

\section{CONFLICT OF INTEREST}

The authors confirm that this article content has no conflict of interest.

\section{ACKNOWLEDGEMENTS}

Declared none.

\section{REFERENCES}

[1] Hoffman JIE, Kaplan S. The incidence of congenital heart disease. J Am Coll Cardiol 2002; 39: 1890-900.

[2] Kanter RJ, Papagiannis J, Carboni MP, Ungerleider RM, Sanders WE, Wharton JM. Radiofrequency catheter ablation of supraventricular tachycardia substrates after mustard and senning operations for d-transposition of the great arteries. J Am Coll Cardiol 2000; 35: 428-41.

[3] Bae E-J, Noh C-I, Choi J-Y, et al. Late occurrence of adenosinesensitive focal junctional tachycardia in complex congenital heart disease. J Interv Card Electrophysiol 2005; 12: 115-22.

[4] Saoudi N, Cosío F, Waldo A, et al. A classification of atrial flutter and regular atrial tachycardia according to electrophysiological mechanisms and anatomical bases; a Statement from a Joint Expert Group from The Working Group of Arrhythmias of the European Society of Cardiology and the North American Society of Pacing and Electrophysiology. Eur Heart J 2001; 22: 1162-82.

[5] Triedman JK, Saul JP, Weindling SN, Walsh EP. Radiofrequency ablation of intra-atrial reentrant tachycardia after surgical palliation of congenital heart disease. Circulation 1995; 91: 707-14.

[6] Haines DE, DiMarco JP. Sustained intraatrial reentrant tachycardia: clinical, electrocardiographic and electrophysiologic characteristics and long-term follow-up. J Am Coll Cardiol 1990; 15: 1345-54.

[7] Wells R, Khairy P, Harris L, Anderson CC, Balaji S. Dofetilide for atrial arrhythmias in congenital heart disease: a multicenter study. Pacing Clin Electrophysiol 2009; 32: 1313-8.

[8] Feltes TF, Friedman RA. Transesophageal echocardiographic detection of atrial thrombi in patients with nonfibrillation atrial tachyarrhythmias and congenital heart disease. J Am Coll Cardiol 1994; 24: $1365-70$

[9] Garson A, Bink-Boelkens M, Hesslein PS, et al. Atrial flutter in the young: a collaborative study of 380 cases. J Am Coll Cardiol 1985; 6: 871-8.

[10] Yap S-C, Harris L, Silversides CK, Downar E, Chauhan VS. Outcome of intra-atrial re-entrant tachycardia catheter ablation in adults with congenital heart disease: negative impact of age and complex atrial surgery. J Am Coll Cardiol 2010; 56: 1589-96.

[11] Triedman JK, Alexander ME, Love BA, et al. Influence of patient factors and ablative technologies on outcomes of radiofrequency ablation of intra-atrial re-entrant tachycardia in patients with congenital heart disease. J Am Coll Cardiol 2002; 39: 1827-35.

[12] $\mathrm{Wu} J$, Pflaumer A, Deisenhofer I, et al. Mapping of intraatrial reentrant tachycardias by remote magnetic navigation in patients with d-transposition of the great arteries after mustard or senning procedure. J Cardiovasc Electrophysiol 2008; 19: 1153-9.

[13] Ernst S, Babu-Narayan SV, Keegan J, et al. Remote-controlled magnetic navigation and ablation with $3 \mathrm{D}$ image integration as an alternative approach in patients with intra-atrial baffle anatomy. Circ Arrhythm Electrophysiol 2012; 5: 131-9.
Rhodes LA, Walsh EP, Gamble WJ, Triedman JK, Saul JP. Benefits and potential risks of atrial antitachycardia pacing after repair of congenital heart disease. Pacing Clin Electrophysiol 1995; 18: 1005-16.

[15] Mavroudis C, Backer CL, Deal BJ, Johnsrude C, Strasburger J. Total cavopulmonary conversion and maze procedure for patients with failure of the Fontan operation. J Thorac Cardiovasc Surg 2001; 122: 863-71.

[16] Marcelletti CF, Hanley FL, Mavroudis C, et al. Revision of previous Fontan connections to total extracardiac cavopulmonary anastomosis: A multicenter experience. J Thorac Cardiovasc Surg 2000; 119: 340-6.

[17] Bink-Boelkens MT, Velvis H, van der Heide JJ, Eygelaar A, Hardjowijono RA. Dysrhythmias after atrial surgery in children. Am Heart J 1983; 106: 125-30.

[18] Roos-Hesselink JW, Meijboom FJ, Spitaels SEC, et al. Excellent survival and low incidence of arrhythmias, stroke and heart failure long-term after surgical ASD closure at young age. A prospective follow-up study of 21-33 years. Eur Heart J 2003; 24: 190-7.

[19] Morton JB, Sanders P, Vohra JK, et al. Effect of chronic right atrial stretch on atrial electrical remodeling in patients with an atrial septal defect. Circulation 2003; 107: 1775-82

[20] Roberts-Thomson KC, John B, Worthley SG, et al. Left atrial remodeling in patients with atrial septal defects. Heart Rhythm 2009; 6: 1000-6.

[21] Vecht JA, Saso S, Rao C, et al. Atrial septal defect closure is associated with a reduced prevalence of atrial tachyarrhythmia in the short to medium term: a systematic review and meta-analysis. Heart 2010; 96: 1789-97.

[22] Silversides CK, Siu SC, McLaughlin PR, et al. Symptomatic atrial arrhythmias and transcatheter closure of atrial septal defects in adult patients. Heart 2004; 90: 1194-8

[23] Gatzoulis MA, Freeman MA, Siu SC, Webb GD, Harris L. Atrial arrhythmia after surgical closure of atrial septal defects in adults. $\mathrm{N}$ Engl J Med 1999; 340: 839-46.

[24] Akar JG, Kok LC, Haines DE, DiMarco JP, Mounsey JP. Coexistence of type I atrial flutter and intra-atrial re-entrant tachycardia in patients with surgically corrected congenital heart disease. J Am Coll Cardiol 2001; 38: 377-84

[25] Shah D, Jaïs P, Takahashi A, et al. Dual-loop intra-atrial reentry in humans. Circulation 2000; 101: 631-9.

[26] Magnin-Poull I, De Chillou C, Miljoen H, Andronache M, Aliot E. Mechanisms of right atrial tachycardia occurring late after surgical closure of atrial septal defects. J Cardiovasc Electrophysiol 2005; 16: 681-7.

[27] Snowdon RL, Balasubramaniam R, Teh AW, et al. Linear ablation of right atrial free wall flutter: demonstration of bidirectional conduction block as an endpoint associated with long-term success. J Cardiovasc Electrophysiol 2010; 21: 526-31.

[28] Gatzoulis MA, Balaji S, Webber SA, et al. Risk factors for arrhythmia and sudden cardiac death late after repair of tetralogy of Fallot: a multicentre study. Lancet 2000; 356: 975-81.

[29] Khairy P, Aboulhosn J, Gurvitz MZ, et al. Arrhythmia burden in adults with surgically repaired tetralogy of Fallot: a multiinstitutional study. Circulation 2010; 122: 868-75.

[30] Roos-Hesselink J, Perlroth MG, McGhie J, Spitaels S. Atrial arrhythmias in adults after repair of tetralogy of Fallot. Correlations with clinical, exercise, and echocardiographic findings. Circulation 1995; 91: 2214-9.

[31] Nakagawa H, Shah N, Matsudaira K, et al. Characterization of reentrant circuit in macroreentrant right atrial tachycardia after surgical repair of congenital heart disease: isolated channels between scars allow "focal" ablation. Circulation 2001; 103: 699-709.

[32] Chan DP, Van Hare GF, Mackall JA, Carlson MD, Waldo AL. Importance of atrial flutter isthmus in postoperative intra-atrial reentrant tachycardia. Circulation 2000; 102: 1283-9.

[33] Roten L, Lukac P, DE Groot N, et al. Catheter ablation of arrhythmias in ebstein's anomaly: a multicenter study. J Cardiovasc Electrophysiol 2011; 22: 1391-6.

[34] Flinn CJ, Wolff GS, Dick M, et al. Cardiac rhythm after the Mustard operation for complete transposition of the great arteries. N Engl J Med 1984; 310: 1635-8.

[35] Durongpisitkul K, Porter CJ, Cetta F, et al. Predictors of early- and late-onset supraventricular tachyarrhythmias after Fontan operation. Circulation 1998; 98: 1099-107. 
[36] Collins KK, Love BA, Walsh EP, Saul JP, Epstein MR, Triedman JK. Location of acutely successful radiofrequency catheter ablation of intraatrial reentrant tachycardia in patients with congenital heart disease. Am J Cardiol 2000; 86: 969-74.

[37] Kalman JM, VanHare GF, Olgin JE, Saxon LA, Stark SI, Lesh MD. Ablation of "incisional" reentrant atrial tachycardia complicating surgery for congenital heart disease. Use of entrainment to define a critical isthmus of conduction. Circulation 1996; 93: 50212 .

[38] Gelatt M, Hamilton RM, McCrindle BW, et al. Risk factors for atrial tachyarrhythmias after the Fontan operation. J Am Coll Cardiol 1994; 24: 1735-41.
[39] Takahashi K, Fynn-Thompson F, Cecchin F, Khairy P, del Nido P, Triedman JK. Clinical outcomes of Fontan conversion surgery with and without associated arrhythmia intervention. Int J Cardiol 2009; 137: 260-6.

[40] Khairy P, Poirier N, Mercier L-A. Univentricular heart. Circulation 2007; 115: 800-12

[41] Mandapati R, Walsh EP, Triedman JK. Pericaval and periannular intra-atrial reentrant tachycardias in patients with congenital heart disease. J Cardiovasc Electrophysiol 2003; 14: 119-25.

[42] Triedman JK, Bergau DM, Saul JP, Epstein MR, Walsh EP. Efficacy of radiofrequency ablation for control of intraatrial reentrant tachycardia in patients with congenital heart disease. J Am Coll Cardiol 1997; 30: 1032-8. 